\title{
4 tips for working with caregivers of children with somatic disorders
}

Mandeep Kaur, MD, FAPA

S omatic symptom and related disorders-physical complaints that may or may not be medically explained that are associated with significant distress and impairment—are common in children and adolescents, and are often accompanied by anxiety and depression. ${ }^{1}$ Clinicians are likely to see children with these disorders in emergency departments, consultation services, or outpatient clinics. Common presenting symptoms include abdominal pain, headache, nausea, vomiting, dizziness, and seizures. ${ }^{1}$ Talking to the caregivers of these children can be challenging due to the subjective nature of the illness. In this article, I offer 4 tips for mental health practitioners to consider when working with caregivers of children with somatic disorders.

1. Support. Talk to the child and caregiver individually, and then together. Try to understand the caregiver's concerns and express empathy to establish rapport. Being dismissive of their concerns is not going to help the child. Acknowledge the caregiver's complaints and ask how seriously they feel other clinicians regard their concerns. Ask the caregiver about their perception of their child's health, how frequently they worry about their child's health, and the impact their worries have on their lives and their child's life. Often the caregiver and child must miss out on obligations (eg, work, school, extracurricular activities) due to the child's care and medical appointments.

2. Educate. This may be difficult, particularly when interacting with a caregiver who is convinced that their child is seriously physically sick. The caregiver may feel that involving psychiatry services is discrediting their concerns. Your initial interaction may be to allow the caregiver to express their frustrations toward the primary service. When talking with caregivers, avoid using medical jargon; in some instances, however, it may be necessary to use medical terminology to reassure the caregiver that you know what you are talking about. Be direct, and do not give false hope. These children often undergo extensive medical workup before psychiatry services are involved. To minimize conflicting messages from multiple clinicians who are caring for the same child, review the patient's chart in advance, and maintain constant communication with other clinicians involved in the patient's care.

3. Reassure. When the caregiver finally begins to acknowledge the psychological nature of their child's illness, provide them with reassurance, but avoid emphasizing that the child is medically healthy because any relief caregivers gain from this can quickly fade and worsen their anxiety. Discuss the importance of treating underlying anxiety or depression with

\section{Every issue of CURRent PsYCHIATRY} has its 'Pearls'

\section{Yours could be found here.}

Read the 'Pearls' guidelines for manuscript submission at MDedge.com/ CurrentPsychiatry/page/pearls. Then, share with your peers a 'Pearl' of wisdom from your practice.
Dr. Kaur is an Attending General and Child and Adolescent Psychiatrist, Cone Health Behavioral Health Hospital, Greensboro, North Carolina, and Assistant Professor of Psychiatry, UNC Chapel Hill School of Medicine, North Carolina.

Disclosure

The author reports no financial relationships with any companies whose products are mentioned in this article, or with manufacturers of competing products.

doi: 10.12788/cp.0139

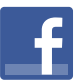

Discuss this article at www.facebook.com/ MDedgePsychiatry 


\section{Referral to a \\ psychotherapist can improve long- term outcomes, especially if coordinated well}

medication and psychotherapy where necessary. Assess the child for substance use disorders, personality disorders, and psychosocial stressors, and if present, target treatment accordingly. Discuss the potential long-term outcomes with and without treatment. Share examples of success stories from your past experiences. Emphasize the importance of noticing even slight improvements. Encourage the child to focus on goals such as attending school or passing online tests, etc.

4. Refer. Connecting the child with a therapist can significantly improve long-term outcomes, especially if coordinated well. ${ }^{2}$ This becomes more crucial in cases where caregivers are opposed to pharmacotherapy for their child. Whenever possible, communicate with the therapist before the child's initial appointment to formulate a plan of action. The best approach is integrated care characterized by close collaboration of primary care, a somatic specialist, and mental health care professionals operating on a biopsychosocial model of distress and therapeutic factors. ${ }^{3}$

The ultimate goal is to help the child and caregiver achieve some level of relief by acknowledgment and support. Utilizing some of these tips can make our work even more meaningful for ourselves and our patients.

\section{References}

1. Malas N, Ortiz-Aguayo R, Giles L, et al. Pediatric somatic symptom disorders. Curr Psychiatry Rep. 2017;19(2):11. doi: 10.1007/s11920-017-0760-3

2. Kurlansik SL, Maffei MS. Somatic symptom disorder. Am Fam Physician. 2016;93(1):49-54.

3. Henningsen P. Management of somatic symptom disorder. Dialogues Clin Neurosci. 2018;20(1):23-31. doi: 10.31887/ DCNS.2018.20.1/phenningsen 